\title{
Social benefits require a community: the influence of colony size on behavioral immunity in honey bees
}

\author{
Rachael E. Bonoan ${ }^{1}$, Paola M. Iglesias Feliciano ${ }^{1,2}$, Joanna Chang ${ }^{1,3}$, \\ Philip T. STARKS ${ }^{1}$ \\ ${ }^{1}$ Department of Biology, Tufts University, 200 College Ave, Medford, MA, USA \\ ${ }^{2}$ College of Natural Sciences, Department of Biology, University of Puerto Rico, San Juan, PR 00931, USA \\ ${ }^{3}$ Department of Biology, Pomona College, 333 North College Way, Claremont, CA, USA
}

Received 22 March 2019 - Revised 14 February 2020 - Accepted 25 February 2020

\begin{abstract}
Emergent properties of eusocial insect colonies (e.g., nest architecture and defense) highlight benefits of group living. Such emergent properties, however, may only function as a benefit if the group is large enough. We tested the effect of group size on colony-level fever in honey bees. When a colony is infected with Ascosphaera apis, a heat-sensitive brood pathogen, adult bees raise the temperature to kill the pathogen and keep brood disease free. In relatively large colonies, we show a rhythm to honey bee fever: colonies inoculated with $A$. apis generated a fever in the afternoon and at night but not in the morning. In comparison, relatively small colonies did not generate a fever following inoculation, although they invested more in thermoregulation on a "per bee" basis than control colonies. Thus, in small colonies, honey bee fever could be regarded as a cost of group living: individuals futilely exerted valuable energy towards fighting a pathogen.
\end{abstract}

group size constraints / behavioral immunity / thermoregulation / sociality

\section{INTRODUCTION}

Group living comes with costs and benefits (Wilson 1975). Some of the most successful group-living organisms on earth are social insects, which exhibit a range of social organization (Wilson 1975). At the pinnacle of social evolution and cooperation are the eusocial insects: individuals work so well together en masse; they can be called a superorganism (Wheeler 1910). In order to successfully function as a superorganism, however, the colony likely needs to host a large enough

Electronic supplementary material The online version of this article (https://doi.org/10.1007/s13592-020-00754-5) contains supplementary material, which is available to authorized users.

Corresponding author: R. Bonoan, rachael.bonoan@tufts.edu

Manuscript editor: James Nieh workforce. This is likely the case when it comes to group-level defense, such as social immunity. For our purposes, we use Meunier (2015)'s definition of social immunity: any collective or individual mechanism that has emerged and/or is maintained at least partly due to the anti-parasite defense it provides to other group members.

Many studies have examined the effect of group size on survival and social immunity in ants and termites. For example, following fungal infection, dampwood termite survival is lower in isolated nymphs than those in groups (Rosengaus et al., 1998). Accordingly, infected dampwood termites show increased immunocompetence and survivorship when raised within a group rather than singly (Traniello et al., 2002). In addition to termites, larger sub-colonies of fungus-infected ants showed quicker rejection of waste and lower mortality rates than their smaller counterparts (Leclerc and Detrain 2018). For these studies, however, 
artificial groups were constructed in the lab. While such studies have added to our understanding of the evolution of sociality in the face of disease, it is also important to consider the natural context of the nest and the social interactions that may occur (Stroeymeyt et al., 2018).

Honey bees (Apis mellifera) are a particularly useful study system to investigate the effect of group size on the success of the superorganism within the context of the nest. First, honey bee population sizes are easy to estimate (see Bonoan et al., 2017), and honey bees can be kept in observation hives for accurate in-nest data collection. Second, honey bee colony sizes vary naturally and exhibit different strengths: Harbo (1986) found that colonies with 35,000 bees produced the most honey (i.e., energy for overwinter survival) per bee, however, colonies with 4500 bees produced the most brood (i.e., reproductive fitness) per bee. In testing different population sizes, Harbo (1986) found 9000 bees to be the optimal colony size for a balance in honey and brood production, or colony survival and reproductive fitness. Third, honey bees act as a superorganism to protect the hive from both macro- and microinvaders via thermoregulation, a behavior that can be easily observed and quantified in observation hives and is likely affected by group size.

When Japanese honey bee (Apis cerana japonica) hives are invaded by giant hornets (Vespa mandarinia japonica), worker bees form a ball around the hornet (Ono et al., 1987; Sugahara and Sakamoto 2009). The worker bees, who can withstand temperatures of up to $48-50{ }^{\circ} \mathrm{C}$ (Coelho 1991), collectively contract thoracic muscles and heat the hornet to a lethal temperature of $45-47^{\circ} \mathrm{C}$ (Ono et al., 1987). While one guard bee initiates the "balling" behavior, it takes a group of 180-300 worker bees to heat the hornet to lethal levels. Adult honey bees also combat micro-invaders via thermoregulation. When the brood is infected with a heatsensitive fungal pathogen, Ascosphaera apis, worker bees use the same contractions of thoracic muscles to actively raise the colony temperature, or generate a behavioral fever (Starks et al., 2000).

A. apis is a brood pathogen that causes a disease known to beekeepers as "chalkbrood" (reviewed in Aronstein and Murray 2010). A temperaturesensitive pathogen, A. apis, enters the larval gut through spores in infected food. Disease symptoms develop only when brood comb temperatures are chilled from the normal 32-36 (Seeley 1995) to $30{ }^{\circ} \mathrm{C}$ or lower (Maassen 1913). If the in-hive temperature is favorable, chalkbrood spores germinate in the hindgut of infected larvae and grow into white hyphae that penetrate the gut and the surrounding tissue (Jensen et al., 2013). Larvae overtaken by this mycelial growth dry out and become chalk-like "mummies" (Maassen 1913; Evison 2015). In response to a chalkbrood infection, worker honey bees generate a behavioral fever by isometrically contracting their thoracic muscles to warm the hive just enough to protect the brood and prevent disease (Starks et al., 2000). For this reason, chalkbrood typically only affects honey bee colonies in the early spring, when ambient temperatures are cooler, or when colonies are already too weak to regulate temperature (Aronstein and Murray 2010).

Here, we use thermal imaging and natural variation in colony size to (1) validate the timing of methods carried out by Starks et al. (2000) to track honey bee fever and (2) investigate efficiency of honey bee fever in large versus small colonies following A apis infection. Similar to what is shown in ants and termites, we hypothesize that larger colonies (i.e., groups) will be able to elicit a behavioral fever, as measured by an increase in in-hive temperature following inoculation, while smaller colonies will not have a large enough workforce to properly function as a superorganism. As such, we predict that larger colonies will generate a successful fever and prevent disease symptoms while smaller colonies will not be able to generate enough heat and ultimately, be overtaken by the disease.

\section{MATERIALS AND METHODS}

\subsection{Study system}

In both 2016 (June) and 2017 (July), eight 2frame observation hives $(53 \mathrm{~cm} \times 48 \mathrm{~cm} \times 5 \mathrm{~cm})$ were filled with honey bees and installed in a temperature-controlled facility at the Starks Lab Apiary on the Tufts University campus in Medford, MA, USA (see Bonoan et al., 2014 for diagram of set-up). All observation hives were queen-right, contained two frames of mixed brood and food, and had similar brood area. After 
installation, the facility was kept at a constant early New England spring-like temperature of approximately $19^{\circ} \mathrm{C}$ and was continuously monitored (HOBO UX100-011 Temperature/Relative Humidity data logger). Following installation, bees were left alone to acclimate to the new location for 3 days. During this time, each colony was fed $150 \mathrm{ml} \mathrm{1:1} \mathrm{sucrose} \mathrm{water.} \mathrm{Upon} \mathrm{inoculation,}$ colonies in 2017 were significantly smaller than in 2016 (ANOVA on LM, $F=9.42, \mathrm{df}=1, p=$ 0.022) (Figure 1).

\subsection{Inoculation with $A$. apis}

Following acclimation, half the colonies $\left(N_{2016}=4, N_{2017}=4\right)$ were fed inoculated 1:2 sucrose water while the other half $\left(N_{2016}=4, N_{2017}=\right.$ 3 ) were fed 1:2 sucrose water without the pathogen. For each inoculum, one chalkbrood mummy (i.e., bee overtaken by the fungus, obtained from the USDA-ARS Bee Research Laboratory) was ground and added to $150 \mathrm{ml} \mathrm{1:2} \mathrm{sucrose} \mathrm{water} \mathrm{(Jensen} \mathrm{et} \mathrm{al.,}$ 2013). All colonies were fed the corresponding sucrose solution in inverted 1-pound queenline jars each day for 3 days. This method was used in both

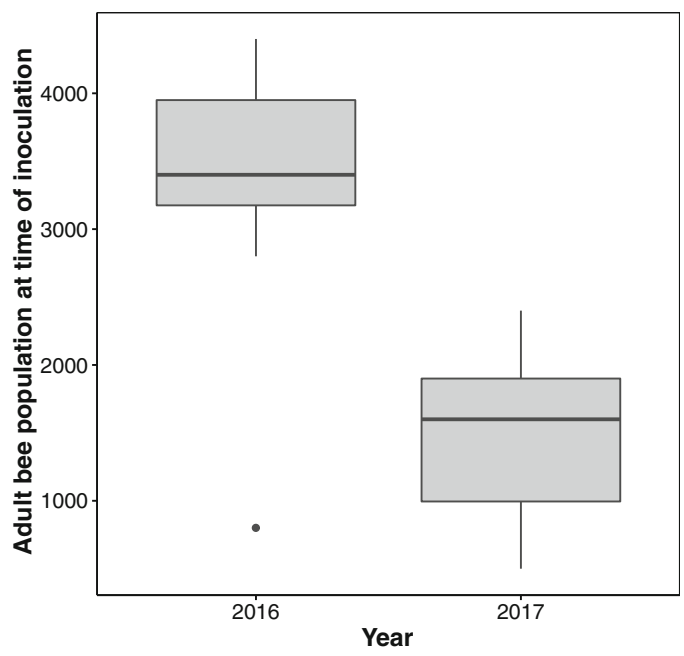

Figure 1. Adult population at the onset of inoculation was in about two times larger in 2016 than in 2017. Gray area shows the range between the first and third quartiles (interquartile range), whiskers denote the lowest and highest values within $1.5 \times$ interquartile range, and the bold black line is the median adult population. $N=8$ colonies in 2016, 7 colonies in 2017 .
2016 and 2017. To carry out field inoculations safely and correctly, we obtained approval (registration \#2016-MIA18) and proper certification from the Tufts University Biosafety Office and worked closely with the Massachusetts Department of Agricultural Resources Apiary Program. To ensure the inoculation was successful, we counted the total number of mummies in each hive on days 4,7 , and 9 post-inoculation (PI) at 08:00 and 16:00.

\subsection{Collection of temperature data and colony size}

In 2016, we used the FLIR ONE (Gen 2 for Android, emissivity $=0.95$ ) personal thermal imager to collect temperature data. Using the FLIR ONE, we recorded the temperature at the central point of each side of each frame in the observation hive for 5day pre-inoculation and ten-day PI. A healthy honey bee colony should develop a colony-level fever (> $30{ }^{\circ} \mathrm{C}$ ) to fight the disease and clean out any mummified larvae within 10 days (Jensen et al., 2013). Following Starks et al. (2000), we collected temperature data at 00:00, 08:00, and 16:00 each day. We estimated the adult population three times a week (Monday, Wednesday, Friday) after thermal imaging at 08:00. Colony size was estimated according to Sammataro and Avitabile (2011): a standard deep frame entirely covered by one layer of bees is roughly 2000 adult individuals, estimates were taken in increments of 250 bees.

In 2017, we used the FLIR E6 thermal imager (emissivity =0.95). Using the FLIR E6, we recorded the average temperature of each side of each frame in the observation hive at 08:00, and 16:00, and 00:00 each day for 3-day pre-inoculation and 10-day PI. We estimated the adult population (see above) every day after thermal imaging at 08:00. In 2017, we used in-hive sensors (BroodMinder) to validate hive surface temperatures determined via thermal imaging as a proxy for internal colony temperature (Supp. Figure 1). In 2019, we used both thermal imaging cameras and protocols to record temperatures of nonexperimental colonies. We confirmed a significant correlation (Spearman correlation, $S=364.28$, $\mathrm{df}=38, p<0.001$, rho $=0.963)$ between the two cameras, and thus, we confidently compare 
temperature data collected in 2016 with data collected in 2017 (Supp. Figure 2).

In both years, the order in which data were collected from each colony was randomized (using random.org to generate random lists) for each collection period.

\subsection{Data analysis}

All analyses were done using car, MASS, plyr, lme4, and glmmTMB in R version 3.3 .2 (201610-31) (R Core Team 2018).

Infection status was confirmed using a zeroinflated generalized linear mixed model (GLMM) with a Poisson distribution in glmmTMB( ) (Brooks et al., 2017) for each year. The models tested for independent and interaction effects of treatment and day PI (mummies $=$ treatment $\times$ day PI).

Colony size in 2016 was compared with colony size in 2017 using a linear mixed model (LM) with fixed effects of year and colony on number of adult bees (number adult bees = year + colony) at the onset of inoculation. To determine significance, we used marginal hypothesis tests, implemented with the Anova() function. Data fit a normal distribution.

In both years, we used the temperature collected during the five (2016) or three (2017) day preinoculation to calculate an average baseline temperature for each colony. We then used this baseline temperature to calculate the mean temperature change from baseline for each colony. Thus, each colony served as its own internal control. We ran LMMs that tested for independent and interaction effects of treatment and day PI on temperature change from baseline (temperature change $=$ treatment $\times$ day PI), with colony added as a random effect. We ran one model for all data pooled, data collected in the morning (08:00), data collected in the afternoon (16:00), and data collected at night (00:00) for a total of four models per year. Again, we used marginal hypothesis tests, implemented with the Anova() function. Data fit a normal distribution.

We also examined the "per bee" effort of warming the hive by dividing the average temperature of the hive by the number of adult bees estimated in the hive that morning. To determine if there was a significant difference in the "degrees per bee", we ran Gaussian family, log link, and generalized linear models (GLMs) that tested for independent and interaction effects of treatment and day PI (degrees per bee $=$ treatment $\times$ day PI). Again, to determine significance, we used marginal hypothesis tests, implemented with the Anova() function. As above, we ran four GLMs: one for all the data together and one for each time of day separately.

\section{RESULTS}

At the onset of inoculation, colonies in 2016 were approximately twice as populous in 2016 (3263 \pm 395 bees) compared with 2017 (1470 265 bees) (Figure 1). Inoculated colonies had significantly more mummies than control colonies in both $2016\left(\mathrm{glmmTMB}, X^{2}=50.19\right.$, df $=1$, $p<0.001)$ and 2017 (glmmTMB, $X^{2}=22.47$, $\mathrm{df}=1, p<0.001$ ) (Figure 2). In both years, hives took longer than 4-day PI to show symptoms of the infection (i.e., mummies).

When all 2016 temperature data were combined, there was a near-significant trend of inoculated colonies exhibiting a higher temperature than control colonies (ANOVA on LMM, $\left.X^{2}=3.56, \mathrm{df}=1, p=0.059\right)$. There was no significant effect of inoculation on temperature change in the morning (ANOVA on LMM, $X^{2}=1.49, \mathrm{df}=1, p=0.222$ ) (Figure 3A). In the afternoon and at night, however, inoculated colonies had significantly higher temperatures than control colonies (afternoon: ANOVA on LMM, $X^{2}=4.38, \mathrm{df}=1, p=0.036$; night: ANOVA on LMM, $X^{2}=4.06, \mathrm{df}=1, p=$ 0.043) (Figure 3B, C). Accordingly, inoculated colonies invested significantly more in stabilizing temperature on a per bee basis than control colonies (Figure 4A-C) (Table 1).

In 2017, when our colonies were on average two times smaller than our colonies in 2016 (Figure 1), we did not detect a fever during any time of the day (Figure 3D-F). Our thermal images showed that our colonies likely did not reach pathogen-killing temperatures: on day-3 PI, we saw one inoculated colony reach $31^{\circ} \mathrm{C}$ at both 00:00 and 16:00, however, the following day, temperature was below $30{ }^{\circ} \mathrm{C}$, and the colony exhibited symptoms of the disease. Although we did not detect a fever in our colonies in 2017, we did find that on a per bee basis, inoculated colonies invested significantly more in stabilizing colony-level temperature (Figure 4D-F) (Table 1). 


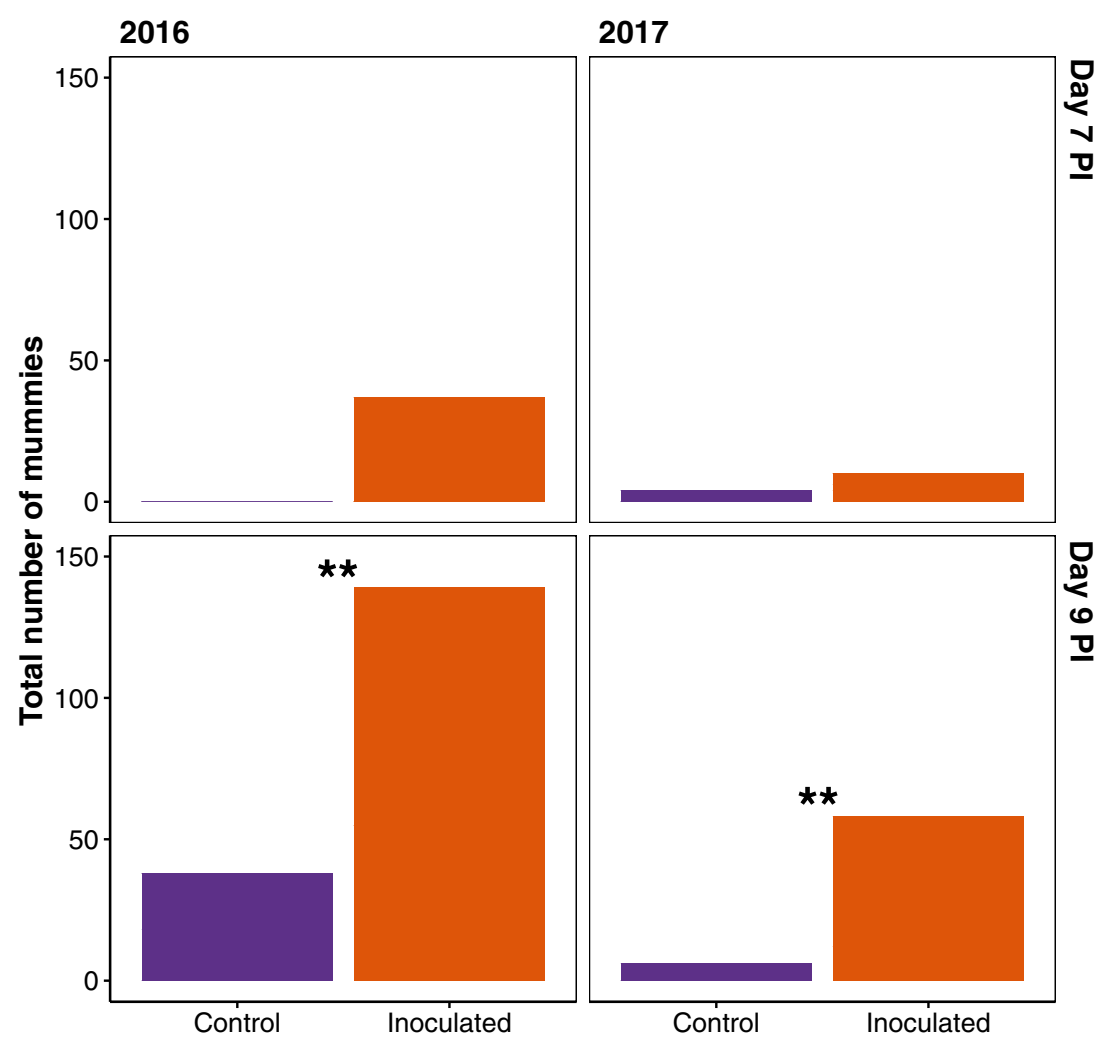

Figure 2. Total number of mummies in inoculated ( $N=4$ for both years) and un-inoculated $\left(N_{2016}=4, N_{2017}=3\right.$ ) hive days 7 and 9 post-inoculation (PI) in 2016 (left) and 2017 (right). In both years, there were no mummies in any hives on day 4 PI, and inoculated hives tended to have more mummies than control hives by day 7 PI with and significantly more mummies by day 9 PI $(* * p<0.001)$.

This was true when all data were analyzed together and for each time point separately. There was also a significant effect of day PI, however, there was no interaction of inoculation and time.

\section{DISCUSSION}

Overall, our data show: (1) a daily rhythm to honey bee fever when colonies are large enough and (2) despite their effort, small colonies are unable to successfully generate an effective colony-level fever.

In 2016, there was no difference in temperature between inoculated and control hives in the morning at 08:00. At 16:00, however, inoculated colonies showed an elevated temperature on days 5-8 PI (Figure 3B), and in the middle of the night at 00:00, inoculated colonies showed an elevated temperature on days 4-6 PI (Figure 3C). While the daily rhythm to honey bee fever may be explained by ambient conditions, we do not feel this is the strongest explanation of the pattern. In order to control for sun exposure and variation in ambient temperature, all observation hives were kept in a temperature-controlled facility and had a small ( $\sim 4 \mathrm{~cm}$ diameter) tube for exiting and entering the hive. Thus, during no time was the sun beating down on the hives themselves. The rhythm of honey bee fever in 2016 is more likely due to the number of bees in the hive, similar to what we see in 2017. During the early summer months when we conducted this study, foraging activity tends to peak in the morning (Rivera et al., 2015). As such, there were likely fewer bees in the hive at 08:00 than later in the day.

In 2017, our colonies did not generate a fever (Figure 3D-F). Although no fever was detected, we did see symptoms of chalkbrood (i.e., mummies) in 


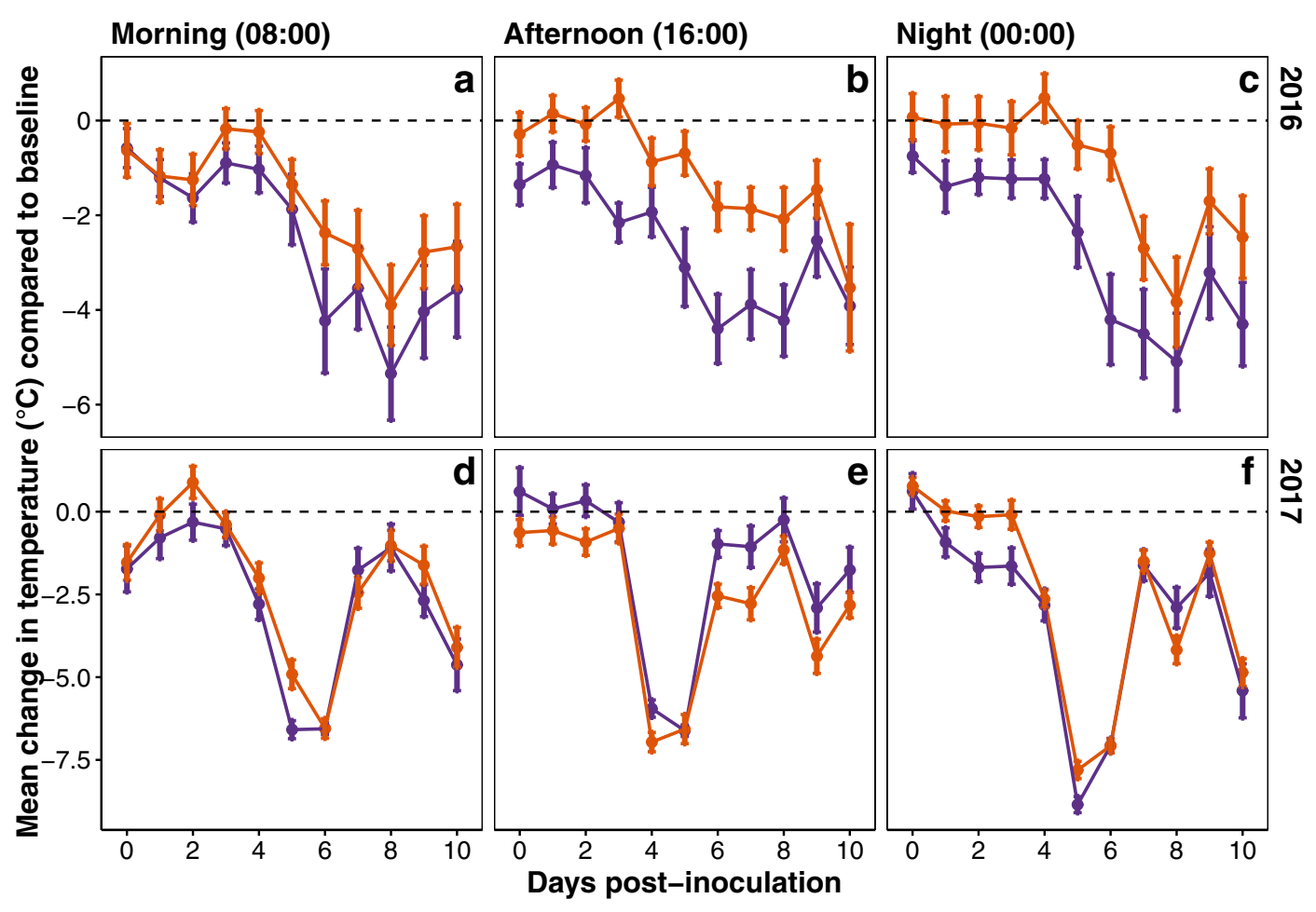

$\rightarrow$ Control $(n=4,3)-$ Inoculated $(n=4,4)$

Figure 3. Mean difference in baseline temperature for control (purple) and inoculated (orange) hives over day PI in 2016 (top panels) and 2017 (bottom panels). Error bars show variation between colonies \pm 1 SE. Data is broken down as follows: (A, D) data collected in the morning; (B, E) data collected at the afternoon; and (C, F) data collected at night. Note: y-axes are different for 2016 and 2017.

the inoculated colonies (Figure 2); therefore, our inoculation procedure was successful. Since we did not see an interaction of inoculation and day PI, the smaller colonies may have already been trying to elevate hive temperature beyond their abilities. There is a significant main effect, however, of day PI: the gap between the two treatments increased as day PI increased (Figure 4). This is likely because the inoculated colonies' population continued to decrease, but remaining workers still attempted to keep temperature stable. Smaller colonies exerted energy trying to fight this heat-sensitive pathogen, even though the outcome was unsuccessful.

Consistent with our data, chalkbrood is known to detrimentally affect honey bee colonies in early spring when ambient temperatures are low or when the colony is already too weak to defend itself (Aronstein and Murray 2010). Since all colonies were kept in the same facility with a controlled ambient temperature, it is likely that our 2017 bees were already too weak to adequately address the experimental inoculation due to the smaller group size. Our data show that this disease, which is normally easily fought via social immunity, disproportionally harms small colonies. This is consistent with data collected by Harbo (1986): different colony sizes resulted in different outcomes.

In addition to group size, our 2016 and 2017 colonies differed in the presence of the parasitic mite, Varroa destructor. There were more sightings (personal observation, REB) of Varroa destructor, a pest known to weaken honey bees and spread disease, in our 2017 colonies than our 2016 colonies. Furthermore, our 2017 colonies exhibited pathological symptoms of a mite infestation, termed Varroasis: unhealthy looking brood (spotty brood pattern, signs of other brood diseases, punctured brood cappings), and adults with deformed wings 


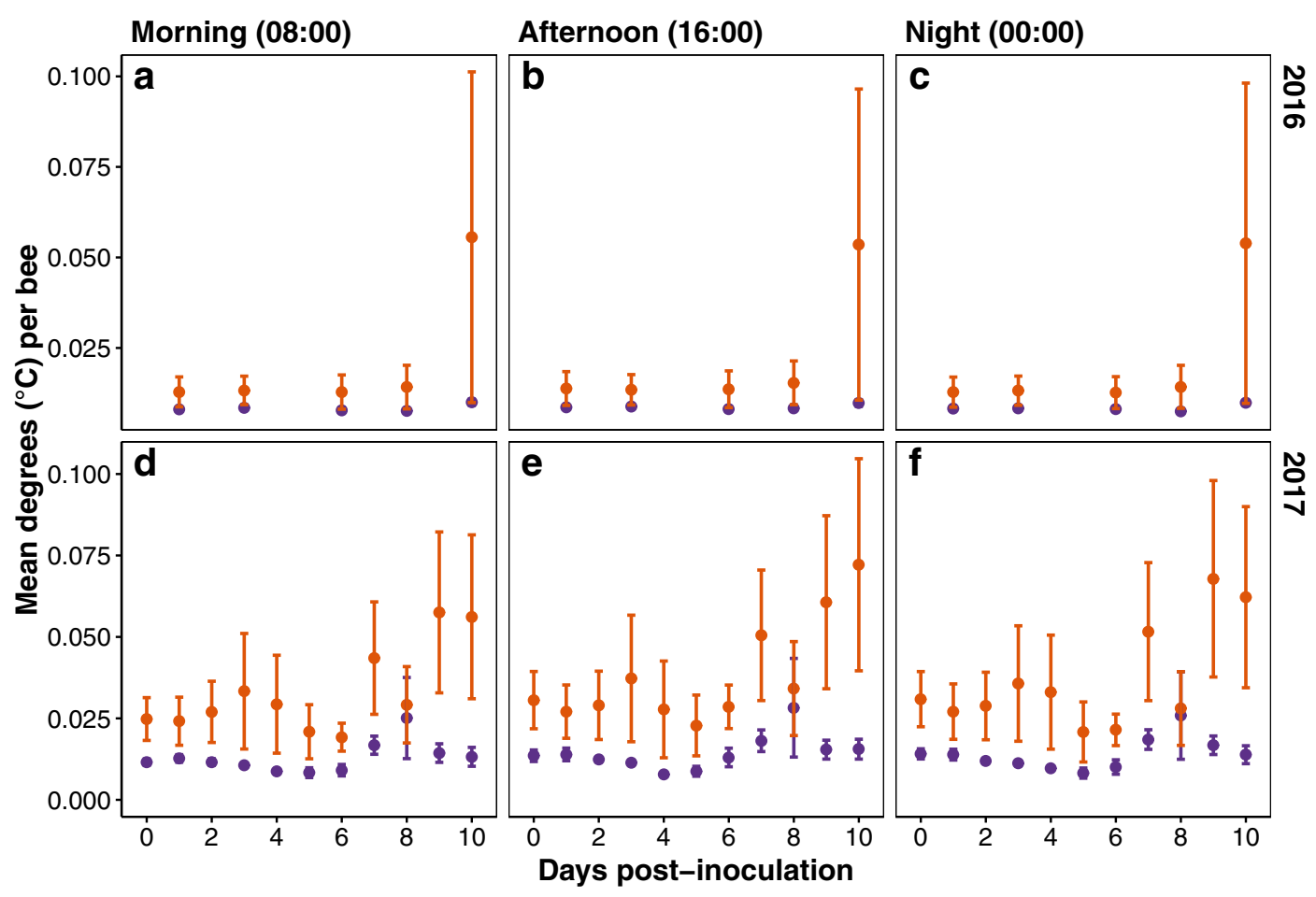

$\rightarrow$ Control $(n=4,3)-$ Inoculated $(n=4,4)$

Figure 4. Mean degree celsius per bee in control (purple) and inoculated (orange) colonies over time in 2016 (top panels) and 2017 (bottom panels). Error bars show variation between colonies $\pm 1 \mathrm{SE}$; dashed line shows the day at which colonies were inoculated. Data is broken down as follows: (A, D) data collected in the morning; (B, E) data collected at the afternoon; and (C, F) data collected at night. Note: y-axes are different for 2016 and 2017.

(Sammataro et al., 2000) which is likely due to the spread of Deformed Wing Virus (DWV). This was true for both inoculated and control hives in 2017. We did not see these symptoms in 2016. Since Varroa mite populations increase in proportion of uncapped larvae, these mites can quickly overrun a colony and cause population die-off.

Our results highlight yet another possible downstream effect of Varroasis: a smaller group made up of weaker adults and thus, the inability to regulate colony temperature. While there is still much to be learned about the effect of DWV on honey bee health, it has been shown that DWV is a driver of overwintering mortality (Highfield et al., 2009). Since honey bees contract their thoracic muscles in order to generate the heat necessary to survive the winter, as they do to generate a behavioral fever, DWV may correlate with thermoregulatory deficiencies. Furthermore, one strain of the virus likely replicates in the thorax of pupal honey bees (Martin and Brettell 2019); among other things, proper development of thoracic muscles is vital to honey bee thermoregulation.

Further studies can use our methods to determine a specific population threshold to honey bee fever. Honey bees exhibit temporal polytheism where younger workers are mainly responsible for tasks inside the hive, such as protecting brood, and older workers are mainly responsible for tasks outside the hive, such as foraging. The distribution of tasks within a colony is affected by both colony demography (Huang and Robinson 1996) and infection status (Lecocq et al., 2016; Stroeymeyt et al., 2018). If there are not enough foragers, younger workers will begin foraging earlier (Huang and Robinson 1996). Similarly, infection with Nosema ceranae, a common fungal pathogen, causes young workers to exhibit behaviors typical of older bees (Lecocq et al., 2016). In both cases, precocious bees are likely not as good at performing tasks as their older counterparts. The same may be true for honey bee fever and 
Table 1. Statistical results for mean degrees per bee. All GLMs tested for fixed effects of treatment, day PI, and the interaction. No interactions were significant

\begin{tabular}{llrrc}
\hline Time of day & Fixed effect & df & $X^{2}$ & \multicolumn{1}{c}{$p$} \\
\hline \multirow{2}{*}{2016} & & & \\
08:00 & Treatment & 1 & 23.93 & $<0.001$ \\
& Days PI & 4 & 30.56 & $<0.001$ \\
\multirow{2}{*}{ 16:00 } & Treatment & 1 & 24.68 & $<0.001$ \\
& Days PI & 4 & 29.27 & $<0.001$ \\
\multirow{2}{*}{$00: 00$} & Treatment & 1 & 23.75 & $<0.001$ \\
& Days PI & 4 & 29.96 & $<0.001$ \\
& & & & \\
\multirow{2}{*}{$08: 00$} & Treatment & 1 & 72.12 & $<0.001$ \\
& Days PI & 10 & 61.42 & $<0.001$ \\
\multirow{2}{*}{ 16:00 } & Treatment & 1 & 75.80 & $<0.001$ \\
& Days PI & 10 & 64.50 & $<0.001$ \\
\multirow{2}{*}{$00: 00$} & Treatment & 1 & 71.08 & $<0.001$ \\
& Days PI & 10 & 68.78 & $<0.001$ \\
\hline
\end{tabular}

thermoregulation in general: with a small population size may come bees that are not necessarily good at their job because they are essentially "filling in."

More broadly, we show evidence for a threshold group size to the proper functioning of this superorganism within the natural context of the colony. As such, in honey bees, defense is a contextdependent benefit to group living. Future studies can use similar methods to investigate the behavioral immune response of various bee species - from eusocial to solitary - to this naturally occurring heat-sensitive pathogen. Such studies can lead to a better understanding of the evolution of sociality and the benefits of group living in bees.

\section{ACKNOWLEDGEMENTS}

We would like to thank James Gonzales, Alexandra Towne, and Adam Winter for help with data collection and beekeeping, Rick Reault of New England Beekeeping for our bees, Jay Evans of the USDA-ARS Bee Research Laboratory for providing the mummies for our inoculums, Kim Skyrm of the Massachusetts Department of Agricultural Resources Apiary Program for ensuring our field inoculations were done safely and correctly, and Heather Mattila and three anonymous reviewers for comments on a draft manuscript.

\section{AUTHOR CONTRIBUTIONS}

REB designed experiments, analyzed data, and wrote the paper. PMIF and JC aided in the data collection and analysis and wrote parts of the paper. PTS aided in experimental design, interpretation, and revision.

\section{FUNDING INFORMATION}

The authors received financial support from the National Science Foundation Research Experience for Undergraduates program at Tufts University (DBI 1560380 to PTS) and Garden Club of America Board of Associates Centennial Pollinator Fellowship (awarded to REB).

\section{OPEN ACCESS}

This article is licensed under a Creative Commons Attribution 4.0 International License, which permits use, sharing, adaptation, distribution and reproduction in any medium or format, as long as you give appropriate credit to the original author(s) and the source, provide a link to the Creative Commons licence, and indicate if changes were made. The images or other third party material in this article are included in the article's Creative Commons licence, unless indicated otherwise in a credit line to the material. If material is not included in the article's Creative Commons licence and your intended use is not permitted by statutory regulation or exceeds the permitted use, you will need to obtain permission directly from the copyright holder. To view a copy of this licence, visit http://creativecommons. org/licenses/by/4.0/.

Le bénéfice social nécessite une communauté : l'influence de la taille de la colonnie sur l'immunité sociale chez les abeilles.

taille des groupes / immunité comportementale / thermorégulation / socialité.

Sozialer Nutzen erfordert eine Gemeinschaft: der Einfluss der Volksstärke auf soziale Immunität bei Honigbienen.

Gruppengröße / Einschränkungen / soziale Immunität / Temperaturregulation / Sozialverhalten. 


\section{REFERENCES}

Aronstein K.A., Murray K.D. (2010) Chalkbrood disease in honey bees. J. Invertebr. Pathol. 103, S20-S29

Bonoan R.E., Goldman R.R., Wong P.Y., Starks P.T. (2014) Vasculature of the hive: heat dissipation in the honey bee (Apis mellifera) hive. Naturwissenschaften 101 (6), 459-465

Bonoan R.E., Tai T.M., Tagle Rodriguez M., Feller L., Daddario S.R. et al (2017) Seasonality of salt foraging in honey bees (Apis mellifera ). Ecol. Entomol. 42 (2), 195-201

Brooks M., Kristensen K., van Benthem K., Magnusson A., Berg C. et al (2017) glmmTMB balances speed and flexibility among packages for zero-inflated generalized linear mixed modeling. R. J. 9 (2), 378-400

Coelho J. (1991) Heat transfer and body temperature in honey bee (Hymenoptera: Apidae) drones and workers. Environ. Entomol. 20 (6), 1627-1635.

Core Team R. (2018) R: a Language and Environment for Statistical Computing. R Foundation for Statistical Computing, Vienna

Evison S.E.F. (2015) Chalkbrood: epidemiological perspectives from the host-parasite relationship. Curr. Opin. Insect Sci. 10, 65-70

Harbo J.R. (1986) Effect of population size on brood production, worker survival and honey gain in colonies of honeybees. J. Apic. Res. 25 (1), 22-29

Highfield A.C., El Nagar A., Mackinder L.C.M., Noël L.M.-L.J., Hall M.J. et al (2009) Deformed wing virus implicated in overwintering honeybee colony losses. Appl. Environ. Microbiol. 75(22), 7212-7220

Huang Z.-Y., Robinson G.E. (1996) Regulation of honey bee division of labor by colony age demography. Behav. Ecol. Sociobiol. 39, 147-158

Jensen A.B., Aronstein K., Flores J.M., Vojvodic S., Palacio M.A. et al (2013) Standard methods for fungal brood disease research. J. Apic. Res. 52 (1), 1-39.

Leclerc J.-B., Detrain C. (2018) Impact of colony size on survival and sanitary strategies in fungus-infected ant colonies. Behav. Ecol. Sociobiol. 72 (1), 2-10

Lecocq A., Jensen A.B., Kryger P., Nieh J.C. (2016) Parasite infection accelerates age polyethism in young honey bees. Sci. Rep. 6, 22042

Maassen A. (1913) Weitere Mitteilungen uber der seuchenhaften Brutkrankheiten der Bienen [further communication on the epidemic brood disease of bees]. Mitt. K. Biol. Anst. Land. u Forstw. 14, 48-58
Martin S.J., Brettell L.E. (2019) Deformed wing virus in honeybees and other insects. Annu. Rev. Virol. 6(1), 49-69

Meunier J. (2015) Social immunity and the evolution of group living in insects. Philos. T. R. Soc. B. 370, 20140102.

Ono M., Okada I., Sasaki M. (1987) Heat production by balling in the Japanese honeybee, Apis cerana japonica as a defensive behavior against the hornet, Vespa simillima xanthoptera (Hymenoptera: Vespidae). Experientia 43 (9), 1031-1034

Rivera M.D., Donaldson-Matasci M., Dornhaus A. (2015) Quitting time: when do honey bee foragers decide to stop foraging on natural resources? Front. Ecol. Evol. 3 (50), 1-9.

Rosengaus R.B., Maxmen A.B., Coates L.E., Traniello J.F.A. (1998) Disease resistance: a benefit of sociality in the dampwood termite Zootermopsis angusticollis (Isoptera: Termopsidae). Behav. Ecol. Sociobiol. 44, 125-134

Sammataro D., Avitabile A. (2011) The Beekeeper's Handbook. Cornell University Press, Ithaca

Sammataro D., Gerson U., Needham G. (2000) Parasitic mites of honey bees: life history, implications, and impact. Annu. Rev. Entomol. 45, 519-548

Seeley T.D. (1995) The Wisdom of the Hive. Harvard University Press, Cambridge

Starks P.T., Blackie C.A., Seeley T.D. (2000) Fever in honey bee colonies. Naturwissenschaften 87, 229-231

Stroeymeyt N., Grasse A.V., Crespi A., Mersch D.P., Cremer S. et al (2018) Social network plasticity decreases disease transmission in a eusocial insect. Science 362 (6417), 941-945

Sugahara M., Sakamoto F. (2009) Heat and carbon dioxide generated by honeybees jointly act to kill hornets. Naturwissenschaften 96 (9), 1133-1136

Traniello J.F.A., Rosengaus R.B., Savoie K. (2002) The development of immunity in a social insect: evidence for the group facilitation of disease resistance. Proc. Natl. Acad. Sci. 99 (10), 6838-6842

Wheeler W.M. (1910) Ants: their Structure, Development and Behavior. Columbia University Press, New York

Wilson E.O. (1975) Sociobiology: the New Synthesis. The Belknap Press of Harvard University, Cambridge, MA, USA.

Publisher's note Springer Nature remains neutral with regard to jurisdictional claims in published maps and institutional affiliations. 